\title{
Individualisierung und Elternschaft - Das Beispiel USA ${ }^{1}$
}

\section{Günter Burkart}

Freie Universität Berlin, Institut für Soziologie, Hittorfstr. 16, D-14195 Berlin

\begin{abstract}
Zusammenfassung: Der Aufsatz knüpft an die deutsche Individualisierungsdiskussion der achtziger Jahre an und versucht, die divergierenden Elemente dieser unübersichtlichen und oft unpräzisen Diskussion in eine überprüfbare Form zu bringen. Das so gewonnene Modell des Individualisierungstheorems wird auf den Bereich Elternschaft bezogen und am Beispiel der amerikanischen Entwicklung der letzten dreißig Jahre empirisch überprüft. Mit der Gegenhypothese „Segmentierung" werden Reichweite und Grenzen der Individualisierungstheorie ausgelotet. Es zeigt sich, da B manche Trends, die gewöhnlich als Indikatoren eines allgemeinen Individualisierungsprozesses interpretiert wurden, entweder anders erklärt werden müssen oder in verschiedenen sozialstrukturellen Zusammenhängen (Milieus) eine unterschiedliche Bedeutung haben. Insgesamt wird die Brauchbarkeit des Individualisierungsbegriffs für die Erklärung familialen Wandels stark in Zweifel gezogen.
\end{abstract}

\section{Die deutsche Individualisierungs- diskussion der achtziger Jahre}

Spätestens mit dem durchschlagenden Erfolg der Risikogesellschaft von Ulrich Beck (1986) hat sich „Individualisierung" zu einer neuen Leitformel der öffentlichen Diskussion innerhalb und außerhalb der Soziologie entwickelt. Innerhalb der Profession blieb die Skepsis groß - zu viele Punkte erschienen fragwürdig. Kaum jemand zweifelt ernsthaft an einem langfristigen Trend dieser Art, doch ist fraglich, ob nach dem Zweiten Weltkrieg tatsächlich ein neuer "Individualisierungsschub" stattgefunden hat. Auch mußte man verwundert zur Kenntnis nehmen, wie schnell scheinbar gesicherte Grundeinsichten der Soziologie, die sich seit den siebziger Jahren auch im öffentlichen Bewußtsein verankert hatten, über Bord geworfen wurden. Auf einmal sollte alles unverbindlich sein, sollten sich alle ganz individuell ihren eigenen Lebensstil patchwork-artig selbst zusammenbasteln können. Von mehreren Seiten wurde außerdem die Behauptung eines universellen Individualisierungstrends in Zweifel gezogen: Manche meinten, es handle sich hier um eine Erscheinung großstädtisch-akademischer Milieus, andere betonten die weiterhin bestehende oder in den achtziger Jahren gar noch gesteigerte Wirksamkeit von Schichtdifferenzen. Schließlich wurde zu Recht kritisiert,

Eine erste Fassung dieses Aufsatzes wurde 1991 während eines von der DFG geförderten Forschungsaufenthalts am Population Studies Center und am Department of Sociology an der University of Pennsylvania, Philadelphia, geschrieben. Frank F. Furstenberg und Susan C. Watkins danke ich für Hinweise und Kritik; ebenso Martin Kohli, Jürgen Wolf, Monika Wohlrab-Sahr und Johannes Huinink. daß die Individualisierungs-Theorie in hohem Maße ungenau und vieldeutig ist. Neben ihrem hohen Allgemeinheitsgrad ist dies der Hauptgrund dafür, daß bisher nur selten der Versuch gemacht wurde, die Theorie in einem strengen Sinn empirisch zu überprüfen. ${ }^{2}$

Als Kernaussage der Individualisierungsdiskussion läßt sich die Behauptung wachsender individueller Wahl- und Entscheidungsmöglichkeiten identifizieren. Sie kann aus einer Reihe von anderen Annahmen abgeleitet werden, deren Zusammenhang sich - stark vereinfacht - wie folgt darstellen läßt: Mit der - u.a. durch Bildungsexpansion und Frauenbewegung - in den letzten Dekaden beschleunigten Freisetzung der Individuen aus traditionalen Bindungen (Klassenmilieu, Familie, Geschlechtsrollen) ist eine Erosion traditioneller Werte - insbesondere Familismus und Versorgungsehe - und eine zunehmende normative Unverbindlichkeit festzustellen. Damit sind Veränderungen auf demographischer, biographischer und HandlungsEbene in Gang gekommen: Eine Pluralisierung von Lebensweisen, insbesondere eine zunehmende Bedeutung individualistischer Lebensstile; wachsende biographische Instabilität (Verlust der biographischen Perspektive der Dauerhaftigkeit, Destandardisierung des Lebenslaufs), aber auch wachsende biographische Autonomie, insbesondere für Frauen (Individualisierung der weiblichen Biographie). Schließlich, als wichtigste Konsequenz auf der Handlungsebene, ein zunehmender Wahl- und Entscheidungsbedarf, der sich aus einer Zunahme biographischer Optionen ergibt. Aller-

2 Empirisch fundierte Kritik z. B. bei Mayer/Blossfeld 1990 sowie weiteren Beiträgen in Berger/Hradil 1990. 
dings sind damit auch „Schattenseiten“ verbunden: Isolation, Vereinsamung, Sicherheitsverlust. ${ }^{3}$ Eine „Überprüfung“ der Individualisierungstheorie (soweit dies überhaupt möglich ist) nach den üblichen Regeln der empirischen Sozialforschung ist ohne aufwendige Primärdatenerhebung nicht durchführbar. Die Ziele dieses Aufsatzes sind bescheidener: Mit Hilfe verfügbarer Daten Reichweite und Grenzen der Kernaussagen des theoretischen Modells auszuloten - beschränkt auf den Bereich „Individualisierung von Elternschaft am Beispiel der USA".

Warum geschieht diese Überprüfung nicht für die Bundesrepublik Deutschland, in der die Individualisierungsdiskussion stattfand, sondern für die Vereinigten Staaten? Zunächst einmal hat die Theorie den Anspruch, die Entwicklung in den fortgeschrittenen Industriegesellschaften der westlichen Welt zu erklären. Allerdings ist bisher in keinem dieser Länder eine ähnliche Diskussion in Gang gekommen. ${ }^{4}$ Es wäre also wünschenswert, die Tragfähigkeit der Theorie an anderen ,westlichen" Ländern zu überprüfen. Die USA haben eine individualistische Tradition, ${ }^{5}$ und eine Reihe von Veränderungen im familialen Bereich, die

3 Die Konstruktion dieses Modells stützt sich hauptsächlich auf folgende Literatur: Beck-Gernsheim 1983, Beck 1986, Zapf et al. 1987, Brose/Hildenbrand 1988, Hoffmann-Nowotny 1988, Herlyn/Vogel 1989, Weymann 1989, Kohli 1989, Heitmeyer/Olk 1990, Beck/Beck-Gernsheim 1990; Meyer 1992.

4 Britische Forscher stehen der Individualisierungstheorie zurückhaltend gegenüber (Jones/Wallace 1990). In einem französischen Lehrbuch über die Familie (Segalen 1990) kommt das Thema nicht vor. Und wenn der Begriff einmal auf amerikanische Verhältnisse angewandt wird (z.B. Buchmann 1989), stößt dies eher auf Skepsis (Sørensen 1990). Gängig ist die Rede vom "family decline" (Popenoe 1988). Shorter (1989) sieht sich bestätigt, daß der von ihm bereits 1975 behauptete Trend zur „postmodernen“ Familie anhält. Ähnlich auch Stacey (1991).

5 Seit Alexis de Tocquevilles Reisebericht über die $D e$ mokratie in Amerika (1835/40) ist es gängig, „Individualismus" als besonderen Charakterzug der amerikanischen Gesellschaft anzusehen (McClosky/Zaller 1984). Historisch verschob sich der Bezug des Begriffs vom furchtlosen Pionier der Siedlerzeit über den politischen Bürger des ausgehenden 18. Jahrhunderts, den Unternehmer des 19. Jahrhunderts (utilitaristisch-ökonomischer Individualismus) schließlich - mit dem Aufstieg des expressiven Individualismus - zum therapeutisch geformten Individuum, das nach Selbstverwirklichung strebt. Neben den Arbeiten von Christopher Lasch ist die Diskussion der letzten Jahre besonders von Habits of the Heart (Bellah et al. 1985) angeregt worden. hierzulande im Zusammenhang mit „Individualisierung" interpretiert werden, waren dort besonders früh oder besonders deutlich zu beobachten, teilweise wesentlich dramatischer: sinkende Fertilität, steigende „Illegitimität”; sinkende Heirats-, steigende Scheidungsquoten; sinkende Haushaltsgrößen, steigende Anteile von singles und cohabitations. $^{6}$

Auf der anderen Seite bieten sich die USA zur Überprüfung der Gegenhypothese an, die annimmt, daß der Individualisierungsproze $\beta$ kein universeller Trend ist (Burkart/Kohli 1989; 1992). Die Vereinigten Staaten sind sozialstrukturell weniger homogen als es die alte Bundesrepublik war, insbesondere in ökonomischer und ethnischer Hinsicht. So könnte es sein, daß auch die Unterschiede zwischen individualisierten Milieus und familistischen Milieus größer sind.

\section{Individualisierung der Elternschaft in den Vereinigten Staaten?}

In diesem Abschnitt wird die These diskutiert, daß der Übergang in die Elternschaft optional geworden sei und im Rahmen biographischer Planung jeweils individuell entschieden werden müsse (,Individualisierung der Elternschaft"). Das allgemeine theoretische Modell der Individualisierung wird auf den Bereich Elternschaft zugeschnitten und überprüft (vgl. Abb. 1). Der Zeitrahmen wird dabei in der Regel auf die letzten drei Jahrzehnte festgelegt, mit der Begründung, daß wesentliche Veränderungen nach 1960 einsetzten. $^{\text {? }}$

\section{Erosion von Werten und Normen hinsichtlich der Normalität und Universalität von Ehe und Familie?}

Der Niedergang des Familismus wird häufig konstatiert. Aber selten wird genauer untersucht, ob ein Zerfall von familialen Normen und Werten festgestellt werden kann. Der familienpolitische Diskurs zeigt die große Unsicherheit der Forschung in

6 International vergleichende Daten und Analysen finden sich zum Beispiel im Heft 3/1988 des Journal of Family Issues sowie bei Sorrentino 1990.

7 In meiner Habilitationsschrift wird diese Überprüfung ausführlicher in Form der Testung einzelner Hypothesen durchgeführt. Auf diese Darstellungsweise wird hier aus Platzgründen verzichtet. Ich danke Stefan Hirschauer für den entsprechenden Vorschlag. 


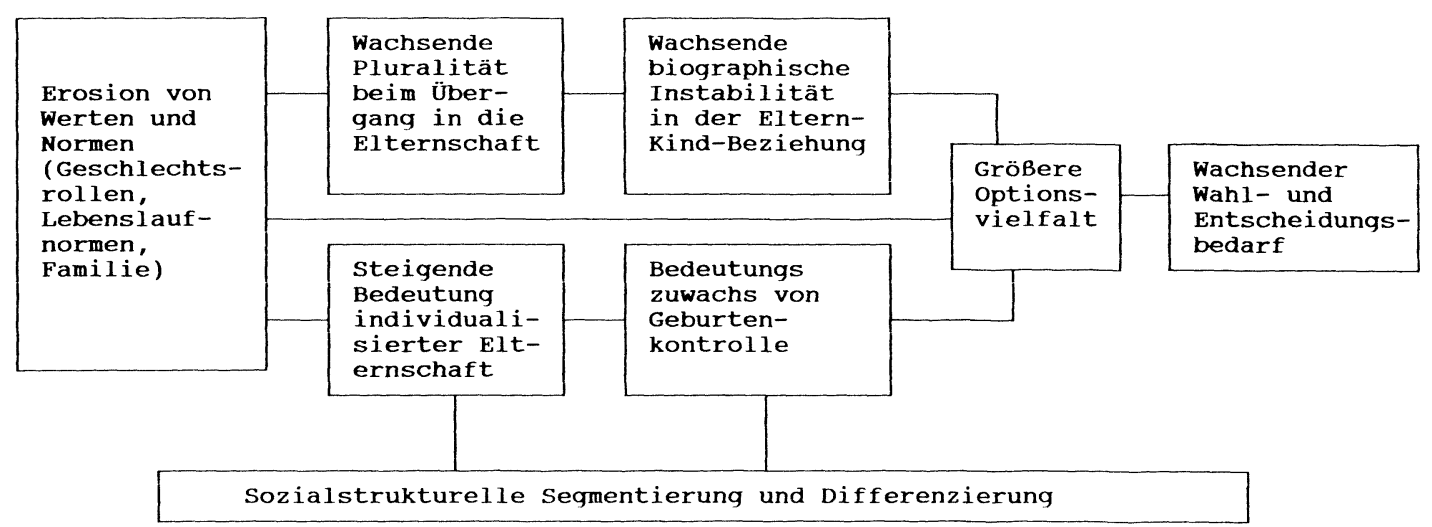

Abb. 1 Elemente von Individualisierung bezüglich Elternschaft und Segmentierung.

dieser Frage: Für manche ist „die Familie“ bereits untergegangen, für andere steht sie weiterhin hoch im Kurs (Berger/Berger 1984, Davis 1985, Cancian 1987, Cherlin 1987, Stacey 1991). Einstellungsdaten geben immerhin Hinweise, daß die Toleranz gegenüber früher als Abweichung definierten Verhaltensweisen gewachsen ist. So zum Beispiel gegenüber Kinderlosigkeit, ebenso gegenüber arbeitenden Müttern oder Frauen, die mit dreißig immer noch unverheiratet sind (Yankelovich 1987, McLaughlin et al. 1988: 169-191, Thornton 1989). Der Familismus als Ideologie ist zwar ebenso geschwächt wie die Ideologie der Frau als Mutter und Hausfrau (Gerson 1985); „Elternschaft“ und „Familie" sind aber dennoch hochbesetzte Werte geblieben (Thornton 1989). Viele Autoren sehen eine Schwächung von Lebenslaufnormen (Altersnormen, Übergangsregeln): Das „script of life" ist nicht mehr so einheitlich - nachdem es in der ersten Hälfte des Jahrhunderts immer homogener geworden war. ${ }^{8}$ Doch es scheint, daß sich neue Normen bilden, zum Beispiel die Erwartung an Frauen, erst eine Ausbildung abzuschließen, bevor geheiratet werden kann. Insgesamt läßt sich konstatieren, daß sich familiale und paarbezogene Werte und Normen gewandelt haben. Für normative Unverbindlichkeit des Verhaltens oder Anomie gibt es jedoch keine klaren Hinweise.

\section{Pluralisierung von Lebensformen?}

Als Konsequenz des normativen Wandels ist auf der demographischen Ebene eine größere Verhal-

8 Modell et al. 1978, Kohli 1985, Modell 1989 zeigen Standardisierungstendenzen; Buchmann 1989 und Cooney/Hogan 1991 solche der Destandardisierung. tensvarianz zu erwarten: „Pluralisierung“ kann als Indikator für Individualisierung angesehen werden, wenn sich zeigen läßt, daß die Spannweite des Verhaltens zunimmt, weil die individuellen Entscheidungsspielräume größer geworden sind. $\mathrm{Zu}$ nächst muß aber geprüft werden, ob die Verhaltensvarianz tatsächlich zugenommen hat. Bezogen auf Elternschaft können drei Punkte unterschieden werden - Pluralisierung würde heißen: a) Die Universalität des Übergangs zur Elternschaft geht verloren, Kinderlosigkeit ist keine seltene Anomalie mehr; b) Die Altersspanne in einer Kohorte beim Übergang zur Elternschaft wächst - es gibt kein Standardalter mehr dafür; c) Die Familiengröße (Zahl der Kinder) ist beliebiger geworden. ${ }^{9}$

a) Der Blick über einen etwas längeren Zeitraum zeigt insbesondere zwei Punkte (vgl. Tab. 1): Das erste bemerkenswerte Ergebnis ist, daß in den Jahrzehnten vor 1960 (1920-1950) der Anteil kin-

9 Daten für die Analyse von Fertilitätstrends in den USA kommen aus mehreren Quellen: 1. Die amtliche Statistik (vital statistics), verwaltet vom U.S. Bureau of the Census und dem Dept. of Health and $\mathrm{Hu}$ man Services. Daten und Analyseergebnisse werden publiziert in jährlichen Statistical Abstracts und Vital Statistics. Diese Daten sind relativ genau und vollständig, disaggregiert allerdings nur für wenige Standard-Variablen. 2. Current Population Surveys (CPS) geben zusätzliche Informationen über Bildung, Eheverlauf oder Geburtsjahr (vgl. Current Population Reports, Series P-20 und P-23). 3. Verschiedene Fertilitäts-Surveys wie die National Surveys of Family Growth (NSFG, seit 1973) erheben ein wesentlich größeres Spektrum sozio-demographischer Faktoren. 4. Verschiedene National Longitudinal Surveys. Für eine Bewertung der Qualität dieser Datenquellen siehe Rindfuss et al. 1988: 4659. 
Tabelle 1 Prozentanteile kinderloser weißer Frauen nach Alter, USA, 1920-1980.

\begin{tabular}{llllllllll}
\hline Alter & 1920 & 1930 & 1940 & 1950 & 1960 & 1965 & 1970 & 1975 & 1980 \\
\hline 20 & 81 & 80 & 82 & 75 & 69 & 73 & 77 & 80 & 81 \\
25 & 45 & 46 & 51 & 36 & 26 & 27 & 35 & 45 & 55 \\
30 & 29 & 28 & 34 & 21 & 15 & 12 & 14 & 20 & 28 \\
35 & 24 & 21 & 24 & 19 & 11 & 11 & 9 & 10 & 15 \\
40 & 23 & 21 & 21 & 21 & 11 & 9 & 9 & 8 & 10 \\
\hline
\end{tabular}

Daten: Census. Quellen: Heuser 1976; Rindfuss et al. 1988: 62.

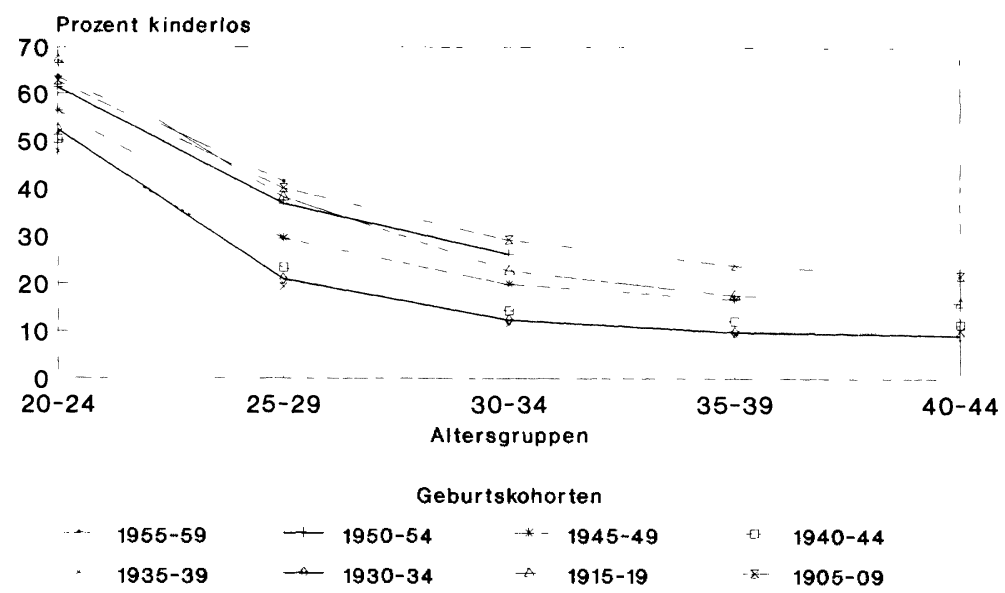

Abb. 2 Kinderlosigkeit nach Altersgruppen und Geburtskohorten von Frauen, USA.

Daten: Current Population Survey. Quelle: U.S. Bureau of the Census, Current Population Reports, Series P-20, No. 421, Fertility of American women: June 1986. Washington, D.C. 1987: 6; Gerson 1985: 236, Tabelle A.5.

derloser Frauen unter den 35- und 40jährigen wesentlich höher war als in den jüngsten Dekaden. Selbst wenn sich als richtig erweisen sollte, daß in den letzten dreißig Jahren der Anteil kinderloser Frauen zugenommen hat - er ist auch 1990 noch nicht so hoch wie fast durchgängig in der ersten Hälfte dieses Jahrhunderts.

Das zweite Ergebnis, das sich unmittelbar aus Tab. 1 ablesen läßt: Kinderlosigkeit hat zwischen 1960 und 1980 zugenommen, doch dies betrifft vor allem die jüngeren Frauen. Das wesentliche Ergebnis ist nicht zunehmende Kinderlosigkeit, sondern biographischer Aufschub der Elternschaft. Bei Tabelle 1 können Perioden- und Kohorteneffekte nicht unterschieden werden. Betrachten wir deshalb Geburtskohorten (Abb. 2). Die Kohorten der ersten beiden Dekaden des 20. Jahrhunderts hatten Anteile von endgültiger Kinderlosigkeit um 20 Prozent. Dann fiel dieser Anteil bis auf etwa 10 Prozent für die in den frühen dreißiger Jahren geborenen Frauen: Sie waren die Mütter des Baby-
Booms. Seither steigt die Kinderlosigkeit von Kohorte zu Kohorte. Der Anteil endgültig kinderloser Frauen liegt bei den jüngsten Kohorten wahrscheinlich bei etwa 15 bis 20 Prozent. ${ }^{10}$

Im Licht der das ganze Jahrhundert durchziehenden Auf- und Abwärtsbewegungen von früher und aufgeschobener Elternschaft bzw. niedriger und hoher Kinderlosigkeit erscheint es zumindest vor-

10 Diese Anteile - je nach Schätzmethode - werden für die Geburtskohorte 1954 von Chen/Morgan (1991) erwartet. Diese Autoren zeigen, daß frühere Schätzungen (z.B. Bloom 1982) für die Kinderlosigkeit jüngerer Kohorten zu hoch waren, weil das Ausmaß des biographischen Aufschubs der Elternschaft damals noch unterschätzt wurde. Die Periodendaten der letzten Jahre zeigen einen Anstieg der Kinderlosigkeit fast in allen Altersgruppen. Zwischen 1980 und 1988 zum Beispiel bei den 35-39jährigen Frauen von $12.1 \%$ auf $17.7 \%$, bei den $40-44$ jährigen von $10.1 \%$ auf $14.7 \%$ (U.S. Bureau of the Census 1989: $10)$. 


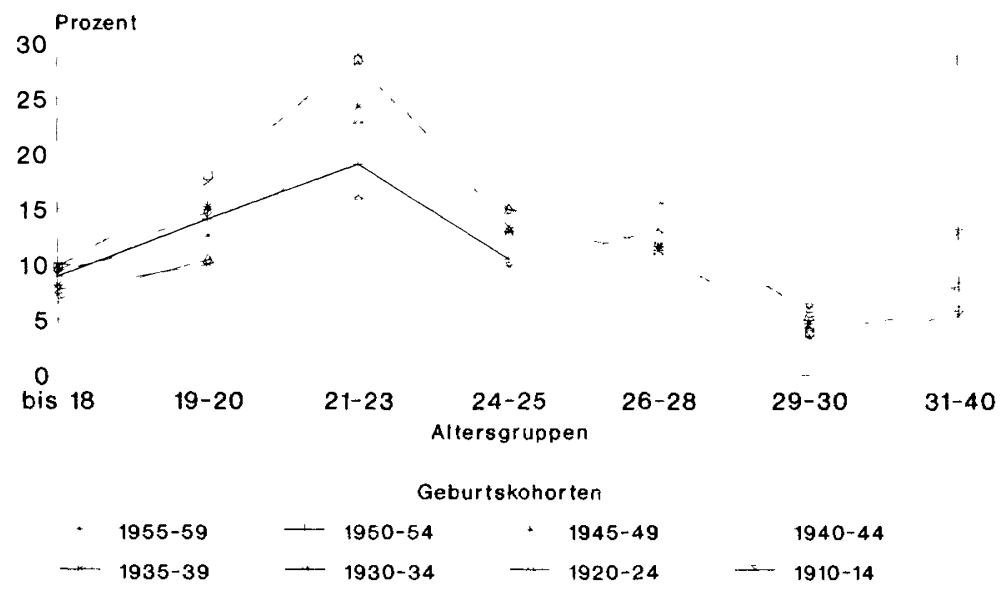

Abb. 3 Übergang in die Elternschaft nach Altersgruppen und Geburtskohorten, USA. Die Prozentuierung bezieht sich auf alle Frauen der jeweiligen Kohorte, nicht nur aut jene, die Kinder bekamen. Daten: Current Population Survey. Quelle: U.S. Bureau of the Census, Current Population Reports, Series P-20, No. 385, Childspacing among birth cohorts of American women: 1905 to 1959. Washington, D.C. 1984: 2.

eilig, den Trend der letzten drei Jahrzehnte als das Ende universeller Elternschaft anzusehen. Dieser Trend ist nicht völlig neu, und für manche Beobachter stellt nicht die hohe Kinderlosigkeit der jüngsten Zeit die Besonderheit dar, sondern die ungewöhnlich niedrige Kinderlosigkeit der Mütter des Baby-Booms. Und einige der führenden amerikanischen Fertilitätsforscher ziehen auch hinsichtlich der zugrundeliegenden sozialen Ursachen Parallelen zwischen den dreißiger und den siebziger/achtziger Jahren. ${ }^{11}$

b) Das durchschnittliche Alter der Mütter bei der Geburt des ersten Kindes stieg von 21.8 im Jahre 1960 auf 23.7 im Jahre 1990. Bedeutet Aufschub der Elternschaft nur Verschiebung der Altersnorm

1 Cherlin 1987; McLaughlin et al. 1988; Rindfuss et al. (1988: 87ff.) zeigen, daß die ökonomischen Probleme in beiden Perioden (in denen die Kohorten der $10 \mathrm{er}$ und $50 \mathrm{er}$ Jahre ins geburtenfähige Alter kamen) ähnlich waren. Es gibt aber noch eine andere, erstaunlichere Parallele: In den beiden Perioden mit hoher Kinderlosigkeit war das Niveau der Bildungsbeteiligung der Frauen besonders hoch (Rindfuss et al. 1988: 118). Morgan (1991) argumentiert, daß die Gründe für die hohe Kinderlosigkeit zu Anfang des Jahrhunderts denen der letzten Dekaden sehr ähnlich seien. Viele Frauen hätten zunächst die Geburt des ersten Kindes aufgeschoben. Aufgrund von Erfahrungen und Umständen sei daraus schließlich zunächst nicht geplante Kinderlosigkeit geworden. oder Auflösung der Altersnorm („Pluralisierung")? Zunächst einmal ist es naheliegend, daß mit der Aufschiebung auch eine gewisse Pluralisierung einhergeht, vor allem, wenn man annimmt, daß längere Ausbildungsphasen für eine wachsende Gruppe von Frauen ein wesentlicher Aufschubfaktor sind (Bloom/Trussell 1984). Doch die Annahme der Auflösung von Altersnormen wird durch die längerfristige Betrachtung erneut relativiert, ähnlich wie für die Untersuchung der Kinderlosigkeit (vgl. Abb. 3). Zwischen den Kohorten von 1910-14 und denen von 1935-39 läßt sich eine zunehmende Homogenität des Alters bei der Geburt des ersten Kindes beobachten. $60 \%$ der Frauen der Kohorten 1935-39 hatten ihre erste Geburt im Alter zwischen 19 und 25. Bei den jüngeren Kohorten nehmen die Anteile der Frauen $a b$, die im jungen Erwachsenenalter ihr erstes Kind bekommen und die Heterogenität steigt wieder. Die älteren und die jüngeren Kohorten ähneln sich: Die Spannweite ist etwas größer, die Kurve ist flacher. Im Jahrhundertvergleich erscheinen die Geburtskohorten der dreißiger/vierziger Jahre als Abweichung, und die Homogenität ihres Übergangs in die Elternschaft in den fünfziger Jahren ist ungewöhnlich.

c) Auch hinsichtlich der Kinderzahl ist keine Pluralisierung nachzuweisen. Im Gegenteil: Es gibt (in der Periodenbetrachtung) eine zunehmende 
Konzentration auf Familien mit ein bis zwei Kindern, die Anteile der größeren Familien nehmen ständig $a b .{ }^{12}$

Zusammengenommen ergeben die drei Teilhypothesen zur wachsenden Variabilität zwischen 1960 und 1990 („Pluralisierung“) folgendes Bild: Der Übergang zur Elternschaft ist weniger universell - Kinderlosigkeit steigt an. Doch er wird immer noch von etwa vier Fünfteln einer Kohorte vollzogen, in der Regel jedoch zu einem späteren biographischen Zeitpunkt als vor dreißig Jahren. Die Altersspannweite ist dabei nur wenig größer geworden. Das Bild der aufgeschobenen Elternschaft und der höheren Kinderlosigkeit der siebziger und achtziger Jahre ähnelt jenem der zwanziger und dreißiger Jahre. Die Homogenität der fünfziger Jahre mit fast universeller Elternschaft und der Konzentration auf das junge Erwachsenenalter erscheint exzeptionell. Hinsichtlich der Familiengröße ist eine stärkere Konzentration auf kleinere Kinderzahlen zu beobachten. Von „Pluralisierung " läßt sich also in einem gehaltvollen Sinn nicht sprechen.

\section{Zunehmende biographische Instabilität - wachsende Bedeutung von Single-Elternschaft?}

Die „vollmobilen Singles“ (Beck, HoffmannNowotny) gelten in der deutschen Diskussion als Inbegriff der neuen Zeit. Alleinleben als Lebensform der Zukunft, die die Familie ablöst. Was bedeutet „Single“ im Hinblick auf die Elternschaft? Sind single-mothers die neuen autonomen Frauen, die auch als Mütter nicht mehr auf die Ehe bzw. einen Mann angewiesen sind, wie in manchen Untersuchungen behauptet (z.B. Eiduson 1980, vgl. Nave-Herz 1992)? 1988 wurden in den USA mehr als neun Millionen Einelternfamilien gezählt (Mutter mit Kind/ern 8,15 Mio., Vater mit Kind/ ern 1,2 Mio.). Zwischen 1970 und 1988 sank der Anteil der Familien mit zwei Eltern von $87.1 \%$ auf

12 So halbierte sich z.B. zwischen 1960 und 1988 bei der Betrachtung von Familien mit eigenen Kindern unter 18 Jahren der Anteil von Drei- und Mehr-KindFamilien von $20.5 \%$ auf $9.8 \%$ (U.S. Bureau of the Census 1961: 39, 1990: 50). Die Analyse der endgültigen Kinderzahl (completed parity distribution) im Kohortenvergleich bestätigt dieses Ergebnis (Gupta 1985). Und für die jüngste Zeit zeigt sich beim Kohortenvergleich in der Altersgruppe 40-44: Die markanteste Veränderung - neben dem weiteren Rückgang großer Kinderzahlen - ist die zunehmende Konzentration auf zwei Kinder (U.S. Dept. of Health and Human Services 1990, section 1: 22).
$72.5 \%$, der Anteil von alleinerziehenden Müttern verdoppelte sich von $11.5 \%$ auf $23.7 \%$, der Anteil alleinerziehender Väter verdreifachte sich von $1.3 \%$ auf $3.6 \%$ (Rawlings 1989). Zweifellos ist dies eine dramatische Veränderung. Doch alle Indizien sprechen dafür, daß es sich hier nicht um „Individualisierung" handelt - weder im Sinne des amerikanischen Verständnisses (Selbstverwirklichung) noch im Sinne der deutschen Diskussion (Zuwachs an Entscheidungsautonomie). Die wenigsten sind single-mother by choice. Die überwiegende Mehrheit der Alleinerziehenden in den Vereinigten Staaten sind geschiedene oder getrenntlebende Mütter, als Folge familiärer Zusammenbrüche. Und ihre ökonomische Situation ist in der Regel außerordentlich schlecht. ${ }^{13}$

Die Problematik der Scheidungsfolgen für Kinder und der Zunahme von Stieffamilien entwickelte sich zu einem der wichtigsten Forschungsgebiete der amerikanischen Familiensoziologie. Da in den meisten Fällen die Kinder bei den Müttern verbleiben, läßt sich festhalten, daß der Übergang zur Elternschaft vor allem für Väter heute keine Gewähr mehr für eine lebenslange Eltern-Kind-Beziehung bietet. Aus der komplementären Perspektive der Kinder gilt entsprechend: Für viele ist die Bindung zu beiden Eltern nicht mehr lebenslang gegeben. Kinder aus zerrütteten Ehen beginnen ihren Lebenslauf mit einer instabilen Situation. „The life course of children of divorce" (Cherlin/Furstenberg 1991: 9) ist bereits eine feste Kategorie der Forschung.

\section{Wachsende biographische Kontrolle?}

Haben wir heute mehr Kontrolle über unser Leben als die Generationen vor dreißig Jahren? Elternschaft kann heute geplant werden, besonders seit der „kontrazeptiven Revolution“ (Westoff/ Ryder 1977) durch die „Pille“. Es könnte also gesagt werden: „Elternschaft ist heute weit stärker als früher das Ergebnis autonomer, bewußter und rationaler Kontrolle über das eigene Leben. Der deutlichste Indikator dafür ist ein höheres Maß an wirksamer Geburtenkontrolle".

13 Es gibt ein für europäische Verhältnisse erstaunlich hohes Maß an Armut unter geschiedenen Frauen (für Männer ist eine Scheidung seltener ein ökonomisches Problem). „Fewer than one custodial mother in four was receiving regular and full child support" (Furstenberg 1990a: 386). Single-Mothers: "No other major demographic group is so poor, and none stays poor longer" (Garfinkel/McLanahan 1986: 167). 
Im Verlauf der sechziger Jahre stiegen die Akzep$\tan z$ und der Gebrauch neuer, wirksamer Kontrazeptiva deutlich an (Westoff/Ryder 1977). Der Anteil unerwünschter Schwangerschaften sank auf knapp 30\% Mitte der achtziger Jahre (Scanzoni/ Scanzoni 1988: 445). Allerdings, so muß man festhalten: Immer noch dreißig Prozent aller Schwangerschaften sind ,unerwünscht”, und für einen wesentlich größeren Teil ist zumindest der Zeitpunkt nicht geplant. Die Bedeutung der „Pille“ für den Zuwachs an Autonomie wird oft überschätzt. Ihre Verbreitung stieg zwischen 1965 und 1976 nur mäBig, sank aber deutlich bei verheirateten Frauen im Verlauf der siebziger Jahre (U.S. Bureau of the Census 1980: 68, 1989: 69). Den größten Zuwachs verzeichnete die Sterilisierung, ein deutliches Zeichen für eine bestimmte Form der Geburtenkontrolle: frühzeitiges Beenden der fruchtbaren Phase. Zumindest in dieser Hinsicht hat die biographische Kontrolle zugenommen. ${ }^{14}$

Aber es gibt auch Anzeichen dafür, daß das Ausmaß an Geburtenkontrolle nicht gewachsen ist. Studien weisen nach, daß bereits in den zwanziger und dreiBiger Jahren ein hohes Maß an Geburtenkontrolle zu registrieren war: Anders läßt sich die außerordentlich niedrige Fertilität der erwachsenen Frauen jener Jahre kaum erklären. ${ }^{15}$ Es gab also auch früher schon mehr Planung, Aufschub, eheliche Fruchtbarkeitskontrolle, usw., als die unvorsichtige Gleichsetzung von ,traditional $=$ kulturelle Selbstverständlichkeit" und „modern = individuelle Entscheidung" nahelegt. Und manche argumentieren, daß die Durchsetzung der „Pille“ keinen Gewinn an bewußter Kontrolle darstellt, da man jetzt nicht mehr „aufpassen“ müsse. Ein weiterer Indikator dafür. daß die biographische Kontrolle nicht wesentlich

14 Unter den verheirateten Frauen, die Geburtenkontrolle praktizierten, stieg zwischen 1973 und 1988 der Anteil der Sterilisation von $24 \%$ auf $49 \%$, der Anteil der ,Pille“ sank von $36 \%$ auf $20 \%$, die Verwendung des Kondoms blieb konstant bei knapp 15\% (Mosher 1990: 199f.).

$1571 \%$ der verheirateten weißen Frauen der Geburtskohorten 1901-10 praktizierten Kontrazeption (Dawson et al. 1980). Das ist eine vergleichsweise hohe Quote. Der Unterschied zu heute ist, daß andere Methoden angewandt wurden. Damals hauptsächlich das Kondom, Irrigation (Spülung) und coitus interruptus (,withdrawal”). - Auch nach Morgan (1991) sind die hohen Anteile von Kinderlosigkeit zu Beginn des Jahrhunderts nur zu erklären, wenn man von erfolgreich praktizierter Empfängnisverhütung ausgeht. Allerdings kann auch er nur indirekt auf Geburtenkontrolle schließen, da genaue Verhaltensdaten fehlen. gewachsen ist, ist der Rückgang der Effizienz der kontrazeptiven Kontrolle in den siebziger und achtziger Jahren, besonders bei Jugendlichen. ${ }^{16}$

\section{Elternschaft als Entscheidung?}

Die Individualisierungstheorie geht von einer wachsenden Vielfalt biographischer Optionen aus. Mehr lebenslaufbezogene Optionen zu haben heißt, sich zwischen biographischen „Pfaden“ entscheiden, an "Gabelungen“ eine Wahl treffen zu müssen. Während früher für die weibliche Biographie die Option „Kinderlosigkeit" nicht bestand, so eine der gängigen Thesen in der Individualisierungsdiskussion, können sich Frauen heute zwischen Mutterschaft und Kinderlosigkeit entscheiden. ${ }^{17}$ Klar ist zunächst, daß die Dominanz der Alleinverdiener-Familie verschwunden ist. Sie fiel zwischen 1940 und 1988 von $70 \%$ auf $20 \%$. Im selben Zeitraum stieg der Anteil der Doppelverdiener-Familie von $9 \%$ auf $40 \%$ und ist heute der häufigste Typus (Hayghe 1990). Im Verlauf der letzten Jahrzehnte, das scheinen diese und andere Daten klar zu bestätigen, haben amerikanische Frauen immer seltener die Option ,mother only" gewählt. Sie sind häufiger erwerbstätig, als Mutter ebenso wie als kinderlose Frau ${ }^{18}$ Aber haben sie wirklich

16 Zelnik/Kantner (1980) analysierten Sexualverhalten, Kontrazeption und Schwangerschaft bei Teenagern in Großstädten in den siebziger Jahren. Sie stellten einen starken Anstieg sexueller Aktivitäten fest. Die Quote der Schwangerschaften stieg, weil die Benutzung der „Pille“ zurückging zugunsten weniger effektiver traditioneller Methoden.

17 Die Elternschaft kann hier, streng genommen, nicht länger isoliert betrachtet werden; es müßten mehrere Variablen kombiniert werden, um eine komplexe Kategorie Lebenslaufoption zu erhalten: Ehe- und Familienstatus, Lebensform, Berufsstatus. Die Hypothese der Individualisierungstheorie würde dann etwa lauten: Um 1960 gab es für die meisten Paare nur eine Option: Die Alleinversorger-Familie. Für die Frauen also: Hausfrau und Mutter. Heute haben Frauen mehrere Wahlmöglichkeiten: Mutter und Hausfrau, kinderlose Erwerbsfrau, erwerbstätige Mutter. Dazu kommen die Optionen unverheiratet zu bleiben und alleine zuleben.

18 Zum Beispiel fiel der Anteil der nichterwerbstätigen Mütter in der Altersgruppe 35-39 von 45\% im Jahre 1967 auf $23 \%$ im Jahre 1982 . Im selben Zeitraum stieg der Anteil berufstätiger Mütter von $41 \%$ auf $58 \%$ (McLaughlin et al. 1988, Tabelle D-3). Die Unterschiede in der Erwerbsbeteiligung von ledigen Frauen, verheirateten Frauen und Frauen mit Kindern sind nur noch minimal. Selbst Mütter von Kleinkindern haben Erwerbsquoten um $60 \%$ (U.S. Bureau of the Census 1990: 384, 385; Menaghan/Parcel 1990). 
zwischen Optionen gewählt? Haben sie eine biographische Entscheidung getroffen?

Erstaunlicherweise findet man kaum klare empirische Belege für diese These. Die Neue Haushaltsökonomie und Rational-Choice-Theorien arbeiten mit der Unterstellung von individuell-rationalen Entscheidungen, aber die soziale Struktur des Entscheidungsprozesses wird dabei in der Regel nicht untersucht, und empirische Belege über historische Veränderungen werden kaum erbracht. Die Kosten-Nutzen-Terminologie ist weit verbreitet, wenn von der „Entscheidung zur Elternschaft“ die Rede ist (Scanzoni/Szinovacz 1980, Fox 1982, Scanzoni/ Scanzoni 1988: 422ff., Seccombe 1991) - aber es handelt sich dabei in der Regel um Denkmodelle oder fiktive Fallstudien. Niemand hat bisher empirisch gezeigt, daß Paare sich „entscheiden“, Eltern zu werden, nachdem sie „Kosten“ und „Nutzen“ kalkuliert haben. ${ }^{19}$ Auch in der Frauenforschung wird häufig mit der Annahme gearbeitet, daß Frauen heute größere Autonomie besitzen und Elternschaft nicht mehr „Schicksal“ ist. Doch auch zum Beispiel die Studie von Gerson (1985) untersucht nicht, wie der Titel - Hard Choices - erwarten läßt, den Entscheidungsprozeß, sondern arbeitet die Bedingungen heraus, unter denen Frauen typischerweise in verschiedene biographische Pfade gelenkt werden. Gerson zeigt strukturelle Zwänge auf, die für oder gegen Elternschaft, für einen frühen oder einen späten Zeitpunkt des Übergangs, wirksam sind. Und neben diesem Hinweis auf strukturellen Zwang gibt es zwei weitere Argumente gegen die Behauptung der gewachsenen Entscheidungsfreiheit hinsichtlich Elternschaft: Das eine ist, daß auch in der Vergangenheit wahrscheinlich ein höheres Maß an individueller Entscheidungsfreiheit vorhanden war als wir häufig annehmen, wenn wir an den Typus traditioneller Elternschaft denken (Imhof 1981, Linde 1984, Macfarlane 1986). Elternschaft als Schicksal mit der Konsequenz einer unkontrolliert hohen Fertilität hat es in keiner Kultur jemals gegeben.

Das zweite Argument: Bei den wenigen Studien, die es zu dieser Frage gibt, zeigte sich, daß die Geburt des ersten Kindes und der biographische Zeit-

19 Zumindest in einem weit verstandenen Sinn von „Kosten und Nutzen“ hat der Geburtenrückgang natürlich viel damit zu tun, daß Kinder ,teurer“ (in der alten Bedeutung des Wortes: "wertvoller") geworden sind (Zelizer 1985). Diese Grundannahme teilen so unterschiedliche Ansätze wie die Neue Haushaltsökonomie, Rational-Choice-Ansätze, die Individualisierungstheorie, die ,biographische Theorie der Fertilität" (Birg et al. 1991). punkt des Übergangs in die Elternschaft meist keine Konsequenz einer klaren Entscheidung sind. Zwei Grundmodelle sind dabei für die USA charakteristisch: Die sehr jungen Mütter nehmen zunächst das Risiko einer Schwangerschaft in Kauf (indem sie auf sichere kontrazeptive Maßnahmen verzichten), sind dann allerdings zu einer Entscheidung gezwungen - für oder gegen eine Abtreibung (Luker 1975). Die späten Mütter (vielfach Frauen mit College-Ausbildung) müßten in der Lage sein, den richtigen Zeitpunkt abzuwarten, gleichwohl aufzupassen, daß es nicht zu spät wird. ,How late can you wait?" (Menken 1985). Angesichts der Schwierigkeit, sich in dieser Situation richtig zu entscheiden, und angesichts der Tragweite beider Alternativen (Kinderlosigkeit oder Elternschaft) kann man von einer strukturellen Überforderung sprechen - und es ist naheliegend, daß selbst hochgebildete, emanzipierte Frauen sich nicht entscheiden können, sondern die Frage dem Schicksal überlassen: wie ,Monika Seiler", deren Fall wir andernorts dargestellt haben (Burkart/Kohli 1992: 173ff.). ${ }^{20}$

Zusammenfassend kann man sagen: Die positive Antwort auf die Frage, ob man überhaupt Kinder haben will, ist für die meisten amerikanischen Paare nach wie vor selbstverständlich; es gibt kein Entscheidungsproblem. Auch der Zeitpunkt des Übergangs in die Elternschaft wird in der Regel nicht genau geplant. Dem ersten Kind liegt seltener eine Entscheidung zugrunde als in individualistischen Theorien gewöhnlich angenommen wird. Eine wachsende Gruppe entscheidet sich jedoch für den Aufschub der Elternschaft in eine allerdings unbestimmte biographische Zukunft. Viele (etwa 1,5 Mio, pro Jahr legal) entscheiden sich für eine Abtreibung, und viele (etwa 1 Mio. jährlich) entscheiden sich für Sterilisation, nachdem sie die gewünschte Kinderzahl erreicht oder überschritten haben. ${ }^{21}$

20 Etwa ein Drittel der von Veevers (1980) interviewten kinderlosen Frauen hatte sich bereits früh entschieden, kinderlos zu bleiben. Doch für die anderen zwei Drittel war Kinderlosigkeit das Ergebnis eines zunächst geplanten Aufschubs bis "die richtige Zeit" für ein Kind gekommen wäre. Doch diese Zeit kam nie, eine Entscheidung wurde nicht getroffen, irgendwann war klar: Jetzt ist es zu spät. Auch hier ist der Buchtitel (Childless by Choice) zumindest irreführend.

21 Für die USA läßt sich daher - über den Daumen gepeilt - folgende Rechnung aufmachen: Es gibt ungefähr 5,5 Mio. Konzeptionen pro Jahr (errechnet aus: 4 Mio. Geburten und 1,5 Mio. legalen Schwangerschaftsabbrüchen). $30 \%$ der Geburten werden als „unerwünscht" eingestuft (Scanzoni/Scanzoni 1988: 


\section{Grenzen der Individualisierung: Segmentierung und Differenzierung}

Für die bisher untersuchten Annahmen der Individualisierungstheorie konnte nur wenig empirische Evidenz gefunden werden, manche wurden stark in Frage gestellt. An mehreren Stellen wurde bereits darauf hingewiesen, daß manche Annahmen der Theorie nur für bestimmte sozialstrukturelle Gruppen oder Milieus gelten. Dies soll nun systematischer untersucht werden.

Die Grundhypothese, die den Analysen dieses Abschnitts zugrundeliegt, lautet: Der Individualisierungsprozeß läßt sich - wenn überhaupt - nicht in allen sozialen Milieus in gleichem Maße feststellen. Die sozialen Strukturen mancher Milieus bremsen oder verhindern das Aufkommen individueller Wahl- und Entscheidungsfreiheit. Entscheidungen hängen von verfügbaren Optionen $\mathrm{ab}$, die wiederum an strukturelle Bedingungen gebunden sind (Bildung, etc.) und über soziale Faktoren wie Milieu, Stellung im Lebenszyklus, usw. vermittelt werden. Im Gegensatz zu einer der Kernaussagen der (radikalen) Individualisierungstheorie, der zufolge Individualisierung ein Trend ist, der früher oder später alle gesellschaftlichen Schichten und Milieus durchdrungen haben wird, lautet die Prognose dieses Aufsatzes, daß strukturelle Segmentierung weiterhin bestehen bleibt und sich vielleicht sogar verstärkt.

Die Überprüfung dieser Hypothese erfolgt auf zweifache Weise: Erstens durch eine Analyse differentieller Fertilitätstrends bezüglich der Variablen Bildungsgrad und ethnische Herkunft (3.1). Zweitens durch eine Analyse von qualitativen Fallstudien zur Frage der Lösung des Vereinbarkeitskonflikts Beruf - Familie (3.2). ${ }^{22}$

445), das wären 1,2 Mio pro Jahr. Lassen wir einmal unberücksichtigt, daß ein insgesamt höherer Anteil auch "ungeplant" sein dürfte. Addieren wir nun die 1,5 Mio. legalen Abtreibungen (lassen auch hier die nicht erfaßten unberücksichtigt), bei denen wir eine ungeplante Schwangerschaft unterstellen dürfen, und die 1,2 Mio. „unerwünschten" Schwangerschaften, so kommen wir auf 2,7 Mio.: knapp die Hälfte der Schwangerschaften ist demnach ungeplant oder unerwünscht.

22 Die Hypothese des milieuspezifischen Individualisierungstrends wurde an der deutschen Situation entwickelt (vgl. Burkart/Kohli 1989). Für die USA konnte ein solcher Milieuvergleich nicht in gleicher Weise durchgeführt werden.

\subsection{Demographische Trends}

Schon eine flüchtige Analyse offenbart deutliche Unterschiede in der Fertilität und dem Zeitpunkt des Übergangs zur Elternschaft für Frauen mit unterschiedlichen Bildungsgraden und für die Angehörigen verschiedener ethnischer Gruppen. Hier können nur einige Facetten dieses komplexen $\mathrm{Zu}-$ sammenhangs beleuchtet werden. Drei Aspekte sollen betrachtet, drei Hypothesen geprüft werden: 1) das Alter beim Übergang in die Elternschaft, 2) Kinderlosigkeit, 3) Einelternfamilien bzw. Alleinerziehende (single-parents).

\section{1) Übergang in die Elternschaft. Betrachten wir} zunächst die ethnische Variable. Im Kohortenvergleich zeigt sich: Durchgängig im 20. Jahrhundert war der Anteil schwarzer Frauen mit einer Erstgeburt im frühen Alter wesentlich höher als für weiBe Frauen. Schon mit 18 hatten mehr als $20 \%$ der schwarzen Frauen eine erste Geburt - etwa dreimal häufiger als bei den weißen Frauen. Und im Alter von 20 hatten bereits mehr als $40 \%$ aller schwarzen Frauen den Übergang zur Mutterschaft vollzogen - etwa doppelt so viele als bei den weiBen Frauen. Zwar gibt es auch bei den Schwarzen in den letzten Jahren eine deutliche Tendenz zum Aufschub, aber sie fällt schwächer aus als bei den Weißen: Der Unterschied vergrößert sich (vgl. Abb. 4).

Für die Hypothese des starken Zusammenhangs zwischen Bildung und Aufschub der Erstgeburt gibt es eine Vielzahl von Belegen. Tabelle 2 zeigt nicht nur diesen Zusammenhang, sondern darüber hinaus, daß er sich zwischen 1960 und 1980 verstärkte. Schließlich wurde in mehreren Untersuchungen eine starke Korrelation zwischen dem Aufschub der Elternschaft und den beiden Variablen Bildungsgrad und ethnische Zugehörigkeit bestätigt (Wilkie 1981, St. John 1982, Bloom/Trussell 1984, Rindfuss et al. 1988). Und je höher das Bildungsniveau, desto größer ist die Differenz des zeitlichen Aufschubs der Erstgeburt zwischen den ethnischen Gruppen (Rindfuss et al. 1988: 120, 128).

2) Kinderlosigkeit. Im Vergleich der beiden ethnischen Gruppen (weiß/schwarz) zeigt sich, daß Kinderlosigkeit bei den weißen Frauen der jüngeren Kohorten deutlich höher liegt als bei den Schwarzen (Chen/Morgan 1991). Was sind die weiteren strukturellen Variablen von Kinderlosigkeit? Betrachten wir die Altersgruppe der 35-44jährigen Frauen, deren fertile Phase weitgehend abgeschlossen ist. In Tabelle 3 sind einige der Extrem- 


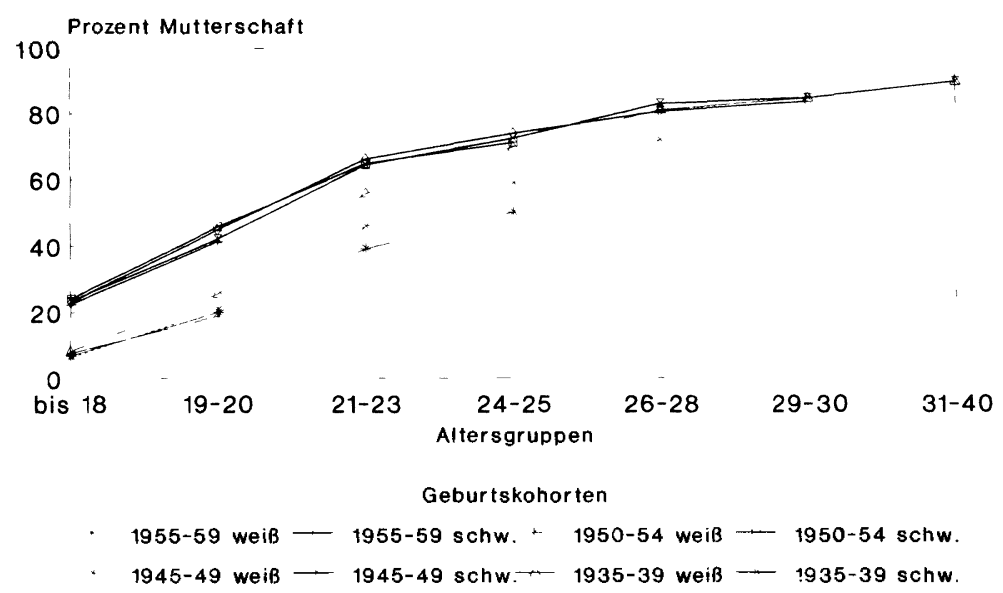

Abb. 4 Übergang in die Elternschaft nach Altersgruppen und ethnischer Zugehörigkeit (Weiß/Schwarz) für ausgewählte Kohorten, USA.

Die Prozentuierung bezieht sich auf alle Frauen der jeweiligen Kohorte, nicht nur auf jene, die Kinder bekamen. Daten: Current Population Survey. Quelle: U.S. Bureau of the Census, Current Population Reports, Series P-20, No. 385, Childspacing among birth cohorts of American women: 1905 to 1959. Washington, D.C. 1984: 2.

Tabelle 2 Aufschub der Elternschaft nach Bildungsdauer, USA, 1960-1980. Durchschnittliche Zeitspanne (in Jahren) von Kinderlosigkeit zwischen dem 18. und 29. Lebensjahr für verheiratete und unverheiratete Frauen nach Länge der Ausbildungszeit (Schuljahre).

\begin{tabular}{|c|c|c|c|c|c|}
\hline \multicolumn{2}{|l|}{ Schuljahre } & 1960 & 1970 & 1980 & $\begin{array}{l}\text { Veränd. } \\
\text { 1960-80 }\end{array}$ \\
\hline \multicolumn{6}{|c|}{ Unverheiratet } \\
\hline \multirow[t]{2}{*}{ weniger als } & 12 & 1.87 & 2.07 & 2.39 & .52 \\
\hline & 12 & 3.00 & 3.07 & 3.85 & .85 \\
\hline mehr als & 12 & 4.97 & 5.20 & 6.42 & 1.45 \\
\hline \multicolumn{6}{|l|}{ Verheiratet } \\
\hline \multirow{2}{*}{ weniger als } & 12 & 1.70 & 1.76 & 1.95 & .25 \\
\hline & 12 & 2.08 & 2.41 & 2.68 & .60 \\
\hline mehr als & 12 & 2.17 & 2.78 & 2.91 & .74 \\
\hline
\end{tabular}

Daten: Current Population Survey.

Quelle: Sweet/Texeira 1984, Tabelle 8.

gruppen (sehr hohe bzw. sehr niedrige Anteile von Kinderlosigkeit) zusammengestellt. Ganz besonders ausgeprägt ist der Zusammenhang von Kinderlosigkeit und Bildungsniveau. Zum Beispiel waren 1986 über $95 \%$ der unverheirateten Frauen mit mindestens 5jähriger College-Ausbildung im Alter von 35-44 (immer noch) kinderlos. (Ihre absolute Zahl ist zwar nicht sehr hoch, aber sie wächst: 187000 im Jahre 1986.) Oder betrachten wir die Gruppe der unverheirateten Frauen in die- ser Altersgruppe in leitenden bzw. professionellen Berufen $(\mathrm{N}=364000): 89.3 \%$ kinderlos. Die wichtigste Variable der Kinderlosigkeit ist dabei allerdings die Ehelosigkeit - mit einer späten Ehe ist deshalb auch eine deutliche Reduzierung der Kinderlosigkeit zu erwarten. Dennoch beeinflußt der Bildungsgrad nicht nur den Aufschub des Übergangs zur Elternschaft, sondern auch die endgültige Kinderlosigkeit bzw. die endgültige Kinderzahl in erheblichem Maße.

3) Single-Parents. Die Individualisierungstheorie erwartet einen deutlichen Anstieg der Zahl „alleinerziehender" Mütter (single-mothers) aufgrund gestiegener Unabhängigkeit der Frauen. Da weiße Frauen mit College-Ausbildung über das größte $\mathrm{Maß}$ an Autonomie verfügen dürften, wäre zu erwarten, daß in dieser Gruppe die Zahl der single-mothers besonders hoch ist. Tabelle 4 zeigt, daß in allen drei ethnischen Gruppen der Anteil der vollständigen Familien zurückgegangen ist. Doch das eindrucksvollste Faktum ist, daß inzwischen die Mehrheit (über $60 \%$ ) der Familien innerhalb der schwarzen Bevölkerungsgruppe ,unvollständig“ ist - während dieser Anteil unter den WeiBen „erst“ bei knapp $23 \%$ liegt. Überwiegend handelt es sich dabei um mother-only families - als Folge von Trennung und Scheidung, aber auch - in weit stärkerem Maß als bei den Weißen - als Folge von nichtehelicher, früher Mutterschaft (vgl. auch Espenshade 1985). 
Tabelle 3 Prozentanteile kinderloser Frauen in der Altersgruppe 35-44 für ausgewählte Gruppen, USA, 1986.

Ausgewählte Gruppen mit hohen Anteilen von Kinderlosigkeit

Ledig, fünf oder mehr Jahre College, weiß

Ledig, Leitende Angestellte und professionelle Berufe, weiß

Ledig, vier Jahre College

91.5

Ledig, Leitende Angestelite und professionelle Berufe

89.3

Ledig, ein bis drei Jahre College

75.0

College, fünt und mehr Jahre

28.6

College, vier Jahre

Ausgewähite Gruppen mit geringen Anteilen von Kinderlosigkeit

Verheiratet, arbeitslos, schwarz

Verheiratet, kein High School Abschluß, hispanische Herkunft

5.9

Verheiratet, Wohnsitz Farm, hispanische Herkunft

7.1

Verheiratet, in Land-, Fisch- oder Forstwirtschaft tätig

7.2

Verheiratet, nicht erwerbstätig, schwarz

7.7

Kein High School Abschluß

9.9

Verheiratet, alle Beschäftigten, schwarz

10.8

Daten: Current Population Survey. Quelle: U.S. Bureau of the Census, Current Population Reports, Series P-20, No. 421, Fertility of American women: June 1986. Washington, D.C. 1987: 14-21.

Tabelle 4 Prozentanteile von Familiengruppen mit Kindern unter 18 Jahren nach ethnischer Zugehörigkeit, USA, 1970-1990.

\begin{tabular}{llcccccccc}
\hline & \multicolumn{3}{c}{ Zwei Eltern } & \multicolumn{3}{c}{ Mutter allein } & \multicolumn{3}{c}{ Vater allein } \\
& 1970 & 1980 & 1990 & 1970 & 1980 & 1990 & 1970 & 1980 & 1990 \\
\hline Weiß & 89.9 & 82.9 & 77.4 & 8.9 & 15.8 & 18.8 & 1.2 & 2.0 & 3.8 \\
Schwarz & 64.3 & 48.1 & 39.4 & 33.0 & 48.7 & 56.2 & 2.6 & 3.2 & 4.3 \\
Hispanisch & n.v. & 74.1 & 66.8 & n.v. & 24.0 & 29.3 & n.v. & 1.9 & 4.0 \\
\hline
\end{tabular}

$n . v .=$ nicht verfügbar. Ethnische Zugehörigkeit des Haushaltsvorstandes. Daten: Current Population Survey. Quelle: U.S. Bureau of the Census, Current Population Reports, Series P-20, No. 447, Household and Family Characteristics: March 1990 and 1989. Washington, D.C. 1990: 7; vgl. auch Rawlings 1989: 13/14.

Widerlegt ist damit die Interpretation von „Alleinerziehenden" als Indikator für Individualisierung (im Sinne größerer Entscheidungsautonomie und Unabhängigkeit von Frauen). Tatsächlich käme wohl niemand in den USA auf den Gedanken, den Anstieg von single-mothers in diesem Sinn zu interpretieren. Die Tatsache, daß Einelternfamilien besonders häufig unter Schwarzen zu finden sind, muß als Indikator für wachsenden sozialen und ökonomischen Problemdruck angesehen werden. Diese Familien gehören zu den Ärmsten der Armen in den Vereinigten Staaten. Andererseits sind sie weniger stark ,individualisiert" als die Weißen: Verwandtschaftliche und nachbarschaftliche Netzwerke spielen bei den schwarzen single-mothers eine weitaus bedeutsamere Rolle als bei den weiBen Frauen (Cherlin 1992). ${ }^{23}$

23 Die statistischen Zahlen vermitteln aufgrund der Besonderheiten der sozialen Situation in den schwarzen Vierteln nur ein unzureichendes Bild über die tatsächliche Situation der unverheirateten Mütter, die in der Regel eben gerade nicht ,alleinerziehend" sind (Stack 1974, Furstenberg et al. 1987). "When is a father really gone?" fragt Mott (1990). Er weist nach, daß die statistisch erfaßte Vater-Absenz eines großen Teils schwarzer Kinder, die mit ihren Mütter leben, die Tatsache verdeckt, daß viele dieser Kinder „either had potentially important substitute figures available or else had significant continuing contact with their biological father" (514). 
Insgesamt ist die Evidenz groß, daß Bildung und ethnische Zugehörigkeit wichtige Faktoren der sozialen Segmentierung sind. Es konnte gezeigt werden, daß einige der Indikatoren, die mit dem Individualisierungstrend in Verbindung gebracht werden, entweder weitgehend auf die gebildete weiße Bevölkerung beschränkt bleiben oder umgekehrt auf Schwarze mit geringem Bildungsniveau konzentriert sind dann aber eine andere Bedeutung haben.

\subsection{Verschiedene Lösungen des Vereinbarkeits- problems Familie - Beruf}

Demographische Daten können zwar Unterschiede im Fertilitätsniveau und im timing des Übergangs zur Elternschaft zwischen verschiedenen sozialen Gruppen aufdecken, sie können jedoch wenig über den Unterschied in der Bedeutung von Elternschaft sagen. Selbst wenn die Unterschiede auf der Ebene der demographischen Daten verschwinden würden, könnten solche Bedeutungsunterschiede bestehen bleiben. Dies ist einer der Gründe dafür, warum der Individualisierungstrend nicht mit demographischen Daten allein überprüft werden kann. Deshalb bezieht sich die weitere Analyse auch auf qualitative Studien; sie konzentriert sich außerdem auf zwei Extremgruppen: Die professionell ausgebildeten weißen Frauen, die selten vor ihrem dreißigsten Lebensjahr (und dies nur unter ganz bestimmten Bedingungen) Mutter werden, auf der einen Seite, und die jungen Mütter aus den schwarzen Siedlungsgebieten auf der anderen Seite.

\section{Kinderlosigkeit und späte Mutterschaft bei}

\section{"Karriere-Frauen"}

Die meisten Frauen arbeiten - auch als Mütter. Aber je höher der Bildungsgrad und je anspruchsvoller die berufliche Tätigkeit, desto schwieriger wird die Realisierung der Mutterschaft (Veevers 1980, Hochschild 1989). Die Studie von Kathleen Gerson (1985) zeigt, von welchen sozialen Bedingungen das Einfädeln in bestimmte biographische Bahnen abhängt, die zur traditionellen HausfrauMutter, zur Kinderlosigkeit oder zur modernen Vereinbarkeitslösung führen. Das traditionelle Modell - Hausfrau und Mutter - stellt für junge Frauen mit College-Ausbildung heute offensichtlich keine Option mehr dar. Kinderlosigkeit war für die Frauen in Gersons Studie keine leichte Entscheidung. Häufig blieben sie kinderlos, wenn sie keinen unterstützenden Partner hatten.
Die Ergebnisse anderer Studien legen den Gedanken nahe, daß die Vereinbarkeit von Beruf und Mutterschaft von finanziellen Ressourcen abhängig ist - manche Doppelkarriere-Paare ermöglichen sich Elternschaft durch private Kinderbetreuung und Haushaltshilfen, typischerweise rekrutiert aus den unteren sozialen Schichten (Hertz 1986; Lehrer/Kawasaki 1985, Grant et al. 1990). Doch es gibt auch Gegenmodelle. Während die Studie von Hertz Frauen vorstellt, die trotz Mutterschaft eine hohe Karriere-Orientierung beibehalten (und ihre ökonomischen Ressourcen einsetzen um häusliche Dienste zu kaufen) hebt eine andere Studie hervor, daß gut verdienende Frauen ihre finanziell günstige Situation nutzen, um ihr berufliches Engagement zu reduzieren, solange die Kinder klein sind (Moen/Dempster-McClain 1987). Dafür spricht auch ein gewisser normativer Druck, den Eltern empfinden, ihre Kinder nicht zu lange in fremde Hände zu geben (Mason/Kuhlthau 1988). Dieser Druck lastet offenbar besonders auf den Frauen: Ergebnisse zweier Studien zum Übergang in die Elternschaft bei jungen Ärzten stellten fest, daß sich die Arbeitszeit der weiblichen Ärzte deutlich reduzierte, wenn sie Mütter wurden, während sich die Arbeitszeit der Väter gewordenen Ärzte sogar leicht erhöhte (Weisman/ Teitelbaum 1987, Grant et al. 1990). Die Autoren dieser Studien führen dies nicht darauf zurück, daß die Männer vor der Familie flüchten (sie beklagten im Gegenteil, zu wenig Zeit für ihre Kinder zu haben), sondern darauf, daß es immer noch einen normativen Druck gibt, der Versorger-Ernährer-Aufgabe zu genügen.

Für Karriere-Frauen, so läßt sich resümieren, gibt es im wesentlichen nur zwei Optionen: Kinderlosigkeit und späte erste Mutterschaft. Die Realisierbarkeit der zweiten Option ist von bestimmten Ressourcen und unterstützenden Bedingungen abhängig.

\section{Junge Mütter}

Die Entkopplung von Sexualität, Ehe und Elternschaft - einerseits frühe Geschlechtsreife und früher Beginn sexueller Aktivitäten, andererseits Aufschub von Ehe und Elternschaft - hat zur Folge, daß viele Frauen heute 15 bis 20 Jahre lang (in Einzelfällen bis zu 30 Jahren) sexuell aktiv sind, bevor sie ihr erstes Kind bekommen. Diese Entwicklung konnte nur problemlos verlaufen, wenn sie von einer sorgfältigen Schwangerschaftsverhütung begleitet war. Doch aus verschiedenen Grün- 
den funktionierte dieser Mechanismus in den Vereinigten Staaten nur unzureichend. Die USA fallen mit ihren hohen Geburtenraten von Teenagern völlig aus dem Rahmen der vergleichbaren westlichen Welt. Das gilt für alle, aber in besonderem Ausmaß für die Schwarzen: Wenn sie erwachsen werden, hat fast die Hälfte aller schwarzen Frauen bereits ein erstes Kind! ${ }^{24}$

Im allgemeinen wird das Phänomen der TeenagerSchwangerschaft als ernsthaftes soziales Problem diskutiert, für dessen Bekämpfung eine Vielzahl von öffentlichen Programmen angeboten wird. Es gibt jedoch auch eine andere Beurteilung. Arline Geronimus (1987) zum Beispiel interpretiert frühe Schwangerschaft als eine kulturell und individuell rationale Antwort auf Deprivation und Diskriminierung. Diesen jungen Frauen, so argumentiert sie, erscheine es vorteilhafter, Mutter zu werden und damit Anerkennung und Integration im Verwandtschafts- und Nachbarschaftsnetz zu erhalten, als die ohnehin geringen Chancen auf eine Ausbildung zu nutzen und gleichzeitig die Nachteile später erster Mutterschaft in Kauf nehmen zu müssen. Gegen diese „rational-choice“-Interpretation früher Schwangerschaft und Mutterschaft spricht jedoch einiges: Die überwiegende Mehrheit dieser Schwangerschaften ist weder gewollt noch geplant. Diese Teenager nehmen das Risiko in Kauf. Sie planen keine Schwangerschaft, aber sie tun auch wenig, sie zu vermeiden (Furstenberg 1990b).

24 Ein Vergleich zwischen mehreren westlichen Ländern ergab, daß amerikanische Teenager in den achtziger Jahren nicht nur die höchsten Geburtenraten, sondern auch die höchsten Schwangerschaftsabbruchraten hatten. Die Schwangerschaftsraten der U.S. Teenager waren mit 96 (auf 1000 Frauen) mehr als doppelt so hoch wie jene von Ländern wie England (45), Canada (44) oder Frankreich (43), fast dreimal so hoch wie jene von Schweden (35) und fast siebenmal so hoch wie jene der Niederlande (14). Selbst die Rate der weißen U.S. Teenager lag mit 83 immer noch mit weitem Abstand an der Spitze (Jones et al. 1985). - Die demographischen Daten sind eindeutig (Miller/Moore 1990, Furstenberg 1990b), die Gründe sind weniger klar. Manche Beobachter erklären die Situation mit der anhaltenden Wirksamkeit der puritanischen Tradition. Öffentliche Werbung für Kondome oder deren kostenlose Verteilung an Schüler zum Beispiel sind bis heute heftig umstritten. Es scheint, daß die amerikanische Öffentlichkeit immer noch eher versucht, die sexuellen Aktivitäten der Jugendlichen zu begrenzen, statt diese zu akzeptieren und für eine effektive Empfängnisverhütung zu sorgen (vgl. Jones et al. 1985, Hayes 1987, Vinovskis 1988).
Wir haben also in den USA zwei Extremgruppen in bezug auf Elternschaft und den Konflikt Beruf Familie, und die Konsequenzen für Lebenschancen und Lebenslauf könnten kaum unterschiedlicher sein. Für die meisten der jungen schwarzen Frauen ist Kinderlosigkeit keine Option. Mutterschaft ist eine Alternative zur kaum zugänglichen Bildungskarriere, und sie steht an der Schwelle zum Erwachsenenalter im Zentrum ihrer Lebensperspektive. Für die weißen College-Frauen ist frühe Elternschaft keine Option. Sie müssen versuchen, erste Mutterschaft und Berufskarriere in einem Lebensalter zu vereinbaren, in dem viele ihrer unterprivilegierten schwarzen Altersgenossinnen bereits Großmutter geworden sind - kaum älter als dreißigjährig! ${ }^{25}$

\section{Theoretische Schlußfolgerungen und offene Fragen}

Die Individualisierungstheorie wurde in mehrfacher Hinsicht in Frage gestellt oder relativiert: Im 2. Abschnitt wurde gezeigt, daß viele Annahmen der Theorie - bezogen auf den Übergang zur Elternschaft in den Vereinigten Staaten von Amerika - nicht bestätigt werden können. Im 3 . Abschnitt wurde gezeigt, daß manche Indikatoren des Individualisierungstrends nicht universell gelten, sondern auf bestimmte Milieus beschränkt bleiben bzw. in verschiedenen Milieus unterschiedlich interpretiert werden müssen. Abschließend sollen nun einige Konsequenzen gezogen und ungeklärte Fragen erörtert werden.

1. „Individualisierung“, so zeigte sich erneut, ist ein unscharfer und vieldeutiger Begriff, mit dem in der deutschen Diskussion zu viele unterschiedliche Phänomene gemeint sind, die bisher nur sporadisch empirisch überprüft wurden und die teilweise sehr unterschiedlich bewertet werden müssen. Mangels einer klaren Vorstellung der empirischen Umsetzbarkeit theoretischer Begriffe wie „Pluralisierung“ oder „Freisetzung“ ist die Individualisierungstheorie bisher nicht operationalisiert worden, und sie verläßt sich - mangels eigens erhobener Daten - manchmal zu sehr auf demographische Daten, ohne genauer auf Bedeutungsunter-

25 Das hat insbesondere Konsequenzen für die Generationenfolge: Man kennt die Extremfälle jener Familiensysteme, auf der einen Seite geprägt durch 30jährige Großmütter und 80jährige Urururgroßmütter; auf der anderen Seite durch eine 75jährige Großmutter, deren einzige Tochter erstmals mit 40 Mutter wurde. 
schiede zu achten. Es ist eine soziologische Binsenweisheit, doch sie muß in diesem Kontext wiederholt werden: Wer statistisch-demographische Daten benutzt, muß sich über die je nach sozialem Kontext sehr unterschiedliche Bedeutung dieser Daten klar sein. Anhänger der Individualisierungstheorie benutzen solche Daten oft unkritisch und deshalb häufig falsch. So wird zum Beispiel der Geburtenrückgang nach 1965 immer überschätzt, wenn man nur auf Perioden-, aber nicht auf Kohortendaten Bezug nimmt. ${ }^{26}$

2. Der Versuch einer Präzisierung der Individualisierungstheorie und ihre Überprüfung am Fall der USA haben gezeigt, daß im Rahmen dieser theoretischen Diskussion sehr viel mehr als bisher auf jeweilige kulturelle Grenzen und Gegentendenzen des behaupteten Individualisierungstrends $\mathrm{zu}$ achten ist. Die meisten der Theorie-Probleme, die am Beispiel der Vereinigten Staaten sichtbar werden, hängen damit zusammen, daß es dort einerseits eine lange Tradition des Individualismus gibt ${ }^{27}$ auf der anderen Seite aber eine Reihe von Gegentendenzen: Trotz der Bedeutung es Individuums waren religiöse und familiäre Bindungen sowie solche auf Gemeindeebene immer recht stark. Darüber hinaus gibt es eine unübersehbare Unterklasse (Wilson 1991); krasse soziale Unterschiede, die den universellen Anspruch der Ideologie individuellen Erfolgs in Frage stellen müßten. Gleichwohl hält sich die Ideologie des Individualismus (,Jeder

${ }^{26}$ Ein anderes notorisches Beispiel ist die Behauptung einer wachsenden Zahl von Singles, die gewöhnlich mit dem wachsenden Anteil der Einpersonenhaushalte begründet wird. Eine einfache Analyse zeigt jedoch, daß dieser grobe demographische Indikator völlig ungeeignet ist, Aufschlu $B$ über die Zahl von Singles zu geben, im wesentlichen aus zwei Gründen: Die Mehrheit der Alleinlebenden sind Witwen und Witwer; und für die Jüngeren ist Alleinleben keine permanente Lebensform, sondern eine begrenzte Übergangsphase im Lebenszyklus - nach dem Auszug aus dem Elternhaus sowie nach Trennung und Scheidung (Burkart 1991). Welchen Sinn macht es, alleinlebende Studenten, postmaritale Singles und Witwen unter das gemeinsame Etikett „Individualisierung" zu packen?

27 Die Siedlermentalität der Kolonialzeit; die früh etablierten demokratisch-liberalen Strukturen und das erfolgreiche wettbewerbsorientierte Wirtschaftssystem des 19. Jahrhunderts; das Fehlen einer starken staatlichen Zentralgewalt ebenso wie das Fehlen einer zentralen Kirche; die ethnische Heterogenität in einem weiten, vielschichtigen Land; das partnerschaftliche Ehemodell (companionate marriage). All das hat Individualisierung und Pluralismus gefördert. ist seines Glückes Schmied“) hartnäckiger als in den Wohlfahrtsstaaten Europas. ${ }^{28}$

3. Manche Entwicklungen von Paarbeziehungen, Ehe und Familie in den Vereinigten Staaten in den letzten drei Dekaden scheinen gut in den Bezugsrahmen der Individualisierungstheorie zu passen, etwa der Aspekt „Destabilisierung des Lebenslaufs". Doch ist gerade im amerikanischen Fall Vorsicht geboten: Was auf den ersten Blick Indikator für Individualisierung zu sein scheint, kann sich bei genauerer Betrachtung als Ausdruck von Segmentierung erweisen. Manche Veränderungen, die hierzulande als Beleg für einen fortschreitenden Individualisierungstrend gesehen werden, traten in den Vereinigten Staaten nicht zuerst in den „,individualisierten Milieus" der Großstädte und akademischen Zentren auf, sondern in der schwarzen Bevölkerung. Die stabile amerikanische Normalfamilie der fünfziger Jahre war eine weiße Mittelklassen-Familie. In der schwarzen Bevölkerung gab es dagegen auch damals schon jene Phänomene, die in den letzten Jahrzehnten die Aufmerksamkeit der Familienforschung auf sich zogen: Unverheiratete Paare, junge ledige Mütter, hohe eheliche Instabilität. Andere Trends, etwa der Anstieg des Heiratsalters oder des Anteils Lediger, haben sich in der schwarzen Bevölkerung in den letzten Jahren stärker beschleunigt als in der weißen (Espenshade 1985, Cherlin 1992). Alle diese Phänomene aber haben bei den Schwarzen mit „Individualisierung " nichts zu tun - was immer man darunter verstehen will. Erklärungen dieser Phänomene müssen an sozialstrukturellen Bedingungen ansetzen: Der Struktur des Verwandtschaftsnetzes, dem Strukturwandel des Heiratsmarktes (zum

28 Man könnte vermuten (anders als Beck oder Zapf et al.), daß Individualisierung durch einen starken Wohlfahrtsstaat gebremst wird, da er Nivellierung fördert. Dem scheint die bekannte schwedische Vorreiterrolle in beiden Dimensionen: Wohlfahrtsstaat und Individualisierung, zu widersprechen. Popenoe (1988) versucht das Paradox mit dem Hinweis darauf zu lösen, daß der schwedische Wohlfahrtsstaat den Individualismus als unbeabsichtigte Nebenfolge fördert, weil die staatliche Bevormundung individuelle Gegenreaktionen geradezu herausfordert. Zunächst einmal gilt, daß sowohl Schweden als auch die USA je spezifische individualistische Traditionen haben. Diese brauchen durch wohlfahrtsstaatliche Maßnahmen nicht notwendigerweise eingeschränkt werden. Auf der anderen Seite sind manche der in Schweden besonders ausgeprägten Züge der familialen Destabilisierung (hohe Ehelosigkeit und hohe „Illegitimitätsraten") keine besonders guten Indikatoren für den Individualisierungstrend. 
Beispiel dem Frauenüberschuß im jüngeren Erwachsenenalter), und nicht zuletzt der anhaltenden Segregation und der wachsenden Armut in den urbanen Ghettos.

Individualisierung, so ein Ergebnis dieses Aufsatzes, ist kein universeller Trend. Er bleibt auf privilegierte soziale Gruppen beschränkt. Insbesondere der Zugang zu höherer Bildung ist - neben der Zugehörigkeit zur weißen Mittelklasse - ein wichtiger Schlüssel für den heute oft konstatierten $\mathrm{Au}$ tonomiezuwachs von Frauen. ${ }^{29}$ Überhaupt, das zeigen viele Analysen, sind Bildungsdifferenzen in den letzten Jahrzehnten nicht verschwunden, sondern im Gegenteil, wichtiger geworden. Das gilt auch für den Heiratsmarkt. Neuere Analysen zeigen, daß zwar religiöse und andere Formen von Homogamie zurückgegangen sind (Schwächung der Heiratsregeln $=$ Individualisierung), die Bildungshomogamie der Ehepaare jedoch größer wurde (Neue Heiratsregel = Segmentierung). Bildung gilt heute als wichtigste soziale Schranke bei der Paarbildung (Mare 1991, Kalmijn 1991).

4. Der Individualisierungsdiskussion fehlt eine mittel- und längerfristige Betrachtung. Nimmt man die fünfziger Jahre als Ausgangspunkt, scheint der Trend in der Tat unübersehbar. Doch besonders die amerikanische Entwicklung läßt auch eine ganz andere Interpretation zu: Die fünfziger Jahre mit ihrer starken Homogenität waren ganz ungewöhnlich (vgl. dazu auch Kaufmann 1988: 402f.), die heutige Situation ähnelt viel mehr jener der dreißiger Jahre. Die Mütter des Baby-Booms der fünfziger Jahre waren die Ausnahme, was den nahezu universellen Übergang in Ehe und Elternschaft in relativ frühem Alter betrifft. Daraus eine Zyklentheorie abzuleiten - in dem Sinne, daß die jüngeren Kohorten in der historischen Wiederholung ähnliche Verhaltensweisen zeigten wie die Kohorten ihrer Großmütter (späte Heirat, hohe Kinderlosigkeit) - ist zwar unsinnig, aber ebenso unhaltbar ist die von der Individualisierungstheorie implizit unterstellte historische Linearität.

29 Deutlich ist der Zusammenhang zwischen Bildungsniveau und Erwerbsquote - und dieser Zusammenhang wurde in den letzten Jahrzehnten noch stärker: Zum Beispiel lag 1988 die Erwerbsquote von Frauen mit weniger als High-School-AbschluB bei $45 \%$, die von Frauen mit mindestens vier Jahren College-Besuch dagegen bei $80 \%$ - ein Unterschied, der noch nie so groß war (U.S. Bureau of the Census 1990: 379). Dic berufliche Segregation nach Geschlecht konnte besonders in jenen Bereichen deutlich abgebaut werden, in denen ein hohes Qualifikationsniveau erforderlich ist (Jacobs 1989).
5. Ein weiteres Problem betrifft den Zusammenhang Individualisierung - Standardisierung. Schon in der Beckschen Fassung des Individualisierungstheorems bleibt das Verhältnis dieser beiden widersprüchlichen Aspekte ungeklärt. Die Rede von der Pluralisierung der Lebensformen, der zunehmenden Optionsvielfalt und der wachsenden Wahlmöglichkeiten ist nicht vereinbar mit Nivellierung und Standardisierung. ${ }^{30}$ Die Individualisierungstheorie hat die individuellen Handlungsspielräume betont - ohne empirisch klären zu können, ob diese wirklich größer sind als früher. Und sie hat die Frage vernachlässigt, welche alten oder neuen Standards für alle oder für einzelne Gruppen und Milieus verbindlich sind. Unterstützt sie damit nicht ungewollt die Ideologie des Individualismus? ${ }^{31}$ Nehmen wir das Beispiel der Geschlechtsrollen. Es gibt manche Hinweise dafür, daß sich die alte Geschlechtsrollendifferenz (wenn auch nur langsam) auflöst. Aber dies kann man unterschiedlich interpretieren. „Individualisierung“ würde heißen: Die Geschlechtsrolle wird unwichtiger für das, was die einzelnen tun und lassen, und jedes Individuum kann sie sich selbst gestalten. "Standardisierung" würde heißen: Männer und Frauen sind zunehmend gezwungen, sich ähnlich $\mathrm{zu}$ verhalten, denselben Erwartungen zu entsprechen. Und ,Segmentierung“ würde heißen: Die universelle Gültigkeit der früheren Geschlechtsrollendifferenzierung löst sich auf, diese wird nun in verschiedenen Milieus neu definiert. Im Alternativmilieu zum Beispiel ist eine Feminisierung der Männer festzustellen, im Akademikermilieu eine Annäherung der Frauen an die männlichen

30) In den USA hat vor allem Parsons immer wieder die Bedeutung universalistischer und generalisierter Orientierungen betont. Auch J.W. Meyer (1986) hat diesen Aspekt der standardisierten Individualität hervorhoben. „In its American variant, individualism is required to be in tune with a highly standardized and universalistic vision of both practical reality and moral purpose. The individual is free, but not to adopt idiosyncratic or parochial purposes ... He is free to expand as a standardized individual ..." (Meyer 1986: 211). Zum Verhältnis von Standardisierung und Individualisierung vgl. auch Kohli 1989 und WohlrabSahr 1992.

31 Zur amerikanischen Ideologie des Individualismus gehört auch die Vorstellung, daß man sich zwar Gruppenstandards anpaßt, aber immer freiwillig, by choice. Und deshalb ist Individualismus die einzige Ideologie, die von Amerikanern akzeptiert wird (Bertelson 1986: 13). Auch Bourdieu hat gezeigt, wie insbesondere im intellektuellen Milieu dic feinen Unterschiede mit der Ideologie des individuellen Unterschieds verknüpft sind. 
Muster (Burkart/Kohli 1989); und schließlich gibt es Milieus, ,wo Männer noch Männer sind“ (Wahl 1991).

6. Was bleibt von der Individualisierungstheorie? Becks Thesen haben eine Fülle wichtiger Fragen aufgeworfen und eine Reihe bemerkenswerter Einsichten gebracht. Aber für die Präzisierung der Theorie-Diskussion hat der „Unbegriff Individualisierung" (Beck 1986: 205) eher geschadet als genützt. Was wir brauchen, sind sorgfältige Analysen soziologisch-demographischer und qualitativer Art, die den Veränderungen im Bereich Paarbeziehung, Ehe und Familie nachgehen ohne sie allzu schnell irgendwelchen theoretischen Schablonen einzufügen; verstärkte theoretische Anstrengungen, insbesondere die Wiederanknüpfung an vernachlässigte Theoriestränge, etwa „funktionale Differenzierung"; genauere Klärungen des Wandels von Werten und Normen, statt diffus von „Werterosion" zu sprechen. Ein vorsichtigerer Umgang mit plakativen Begriffen und gängigen Formeln wie "Individualisierung“, „Pluralisierung“", „Lebensplanung“ oder „rationale Entscheidung“ wäre ratsam. Im Zusammenhang der Renaissance von Rational-Choice-Ansätzen, die ja ebenso wie die Individualisierungstheorie die Entscheidung ins Zentrum rücken, ist eine soziologische Präzisierung des Entscheidungsbegriffs dringend notwendig.

\section{Literatur}

Beck-Gernsheim, E., 1983: Vom „Dasein für andere“ zum Anspruch auf ein Stück ,eigenes Leben“; Individualisierungsprozesse im weiblichen Lebenszusammenhang. Soziale Welt 34: 307-340.

Beck, U., 1986: Risikogesellschaft. Auf dem Weg in eine andere Moderne. Frankfurt: Suhrkamp.

Beck, U./Beck-Gernsheim, E., 1990: Das ganze normale Chaos der Liebe. Frankfurt: Suhrkamp.

Bellah, R.N./Madsen, R./Sullivan, W.M./Swidler, A./ Tipton, S.M., 1985: Habits of the heart. Individualism and commitment in American life. Berkeley: University of California Press.

Berger, B./Berger, P.L., 1984: In Verteidigung der bürgerlichen Familie. Frankfurt: Fischer.

Berger, P.A./Hradil, S. (Hrsg.), 1990: Lebenslagen, Lebensläufe, Lebensstile (Sonderband 7 der Sozialen Welt). Göttingen: Schwartz.

Bertelson, D., 1986: Snowflakes and snowdrifts: Individualism and sexuality in America. Lanham: University Press of America.

Birg, H./Flöthmann, E.-J./Reiter, I.: Biographische Theorie der demographischen Reproduktion. Frankfurt/New York: Campus 1991.
Bloom. D.E., 1982: What's happening to the age at first birth in the United States? A study of recent cohorts. Demography 19: 315-334.

Bloom, D.E./Trussell, J., 1984: What are the determinants of delayed childbearing and permanent childlessness in the United States? Demography 21: 591611.

Brose, H.-G./Hildenbrand, B. (Hrsg.), 1988: Vom Ende des Individuums zur Individualität ohne Ende. Opladen: Leske und Budrich.

Buchmann, M., 1989: The script of life in modern society: Entry into adulthood in a changing world. Chicago: University of Chicago Press.

Burkart, G., 1991: Individualismus und Familialismus. S. 126-129 in: Glatzer, W. (Hrsg.): 25. Deutscher Soziologentag 1990. Die Modernisierung moderner Gesellschaften. Sektionen, Arbeits- und Ad hoc-Gruppen, Ausschuß für Lehre. Opladen: Westdeutscher Verlag.

Burkart, G./Kohli, M., 1989: Ehe und Elternschaft im Individualisierungsproze $\beta$. Bedeutungswandel und Milieudifferenzierung. Zeitschrift für Bevölkerungswissenschaft 15: 405-426.

Burkart, G./Kohli, M., 1992: Liebe, Ehe, Elternschaft: Die Zukunft der Familie. München: Piper.

Cancian, F.M., 1987: Love in America. Gender and selfdevelopment. Cambridge: Cambridge University Press.

Chen, R./Morgan, S.P., 1991: Recent trends in first birth timing in the United States. Paper presented at the 1991 annual meeting of the Population Association of America, Washington, D.C.

Cherlin, A.J., 1987: The trends. Marriage, divorce, remarriage. S. 80-88 in: Skolnick, A.S./Skolnick, J.H. (eds.): Family in transition. Fifth edition. Boston: Little, Brown \& Co.

Cherlin, A.J., 1992: Race and poverty (chapter 4 in:) Marriage, divorce, and remarriage. Revised and enlarged edition. Cambridge (Mass.): Harvard University Press.

Cherlin, A.J./Furstenberg, F.F, 1991: Divided families. What happens to children when parents part? Cambridge: Cambridge University Press.

Cooney, T.M./Hogan, D.P., 1991: Marriage in an institutionalized life course: First marriage among American men in the twentieth century. Journal of Marriage and the Family 53: 178-190.

Dawson, D.A./Meny, D.J./Ridley, J.C., 1980: Fertility control in the United States before the contraceptive revolution. Family Planning Perspectives 12: 76-86.

Davis, K. (ed., 1985): Contemporary marriage. Comparative perspectives on a changing institution. New York: Russell Sage Foundation.

Eiduson, B.T., 1980: Contemporary single mothers. S. 65-76 in: Katz, L.G. (ed.): Current topics in early childhood education. Norwood, N.J.

Espenshade, Th.J., 1985: The recent decline of American marriage. Blacks and whites in comparative perspective. S. 53-90 in: Davis, K. (ed.): Contemporary marriage. Comparative perspectives on a changing institution. New York: Russell Sage Foundation. 
Fox, G.L., 1982: The childbearing decision. Fertility attitudes and behavior. Beverly Hills: Sage.

Furstenberg, F.F., 1990a: Divorce and the American family. Annual Review of Sociology 16: 379-403.

Furstenberg, F.F., 1990b: As the pendulum swings: Teenage childbearing and social concern. Paper, Philadelphia: Population Studies Center.

Furstenberg, F.F./Brooks-Gunn, J./Morgan, S.P., 1987: Adolescent mothers in later life. Cambridge, Mass.: Harvard University Press.

Garfinkel, I./McLanahan, S., 1986: Single mothers and their children. A new American dilemma. Washington, D.C.: Urban Institute Press.

Geronimus, A.T., 1987: On teenage childbcaring and neonatal mortality in the United States. Population and Development Review 13: 245-279.

Gerson, K., 1985: Hard choices: How women decide about work, career, and motherhood. Berkeley/Los Angeles: University of California Press.

Grant, L./Simpson, L.A./Wrong, X.L./Peters-Golden, H., 1990: Gender, parenthood, and work hours of physicians. Journal of Marriage and the Family 52: 39-49.

Gupta, P. das, 1985: Future fertility of women by present age and parity. Analysis of American historical data, 1917-80 (U.S. Bureau of the Census, Current Population Reports, Series P-23, No. 142). Washington, D.C.

Hayes, Ch.D. (ed., 1987): Risking the future. Adolescent sexuality, pregnancy, and childbearing. Panel on adolescent pregnancy and childbearing. Washington, D.C.: National Academic Press.

Hayghe, H.V., 1990: Family members in the work force. Monthly Labor Review 113, No. 3: 14-19.

Heitmeyer, W./Olk, Th. (Hrsg., 1990): Individualisierung von Jugend. Weinheim: Juventa.

Herlyn, I./Vogel, U., 1989: Individualisierung. Eine neue Perspektive auf die Lebenssituation von Frauen. Zeitschrift für Sozialisationsforschung und Erziehungssoziologie 9: 162-178.

Hertz, R., 1986: More equal than others: Women and men in dual-career marriages. Berkeley: University of California Press.

Heuser, R.L., 1976: Fertility tables for birth cohorts by color: Unites States, 1917-73. Washington, D.C.: U.S. Government Printing Office.

Hochschild, A., with Machung, A., 1989: The second shift. Working parents and the revolution at home. New York: Viking.

Hoffmann-Nowotny, H.J., 1988: Ehe und Familie in der modernen Gesellschaft. Aus Politik und Zeitgeschichte, B 13/88: 3-13.

Imhof, A.E., 1981: Die gewonnenen Jahre. Von der Zunahme unserer Lebensspanne seit dreihundert Jahren oder von der Notwendigkeit einer neuen Einstellung zu Leben und Sterben. München: Beck.

Jacobs, J.A., 1989: Revolving doors. Sex segregation and women's careers. Stanford: Stanford University Press.

Jones, E.F. et al., 1985: Teenage pregnancy in developed countries: Determinants and policy implications. Family Planning Perspectives 17: 53-63.
Jones, G./Wallace, C., 1990: Jenseits von Individualisierungstendenzen - Zum aktuellen Wandel der Lebenslaufmuster von Jugendlichen in Großbritannien. S. 125-145 in: Büchner, P./Krüger, H.H./Chisholm, L. (Hrsg.): Kindheit und Jugend im interkulturellen Vergleich, Opladen: Leske + Budrich.

Kalmijn, M., 1991: Shifting boundaries: Trends in religious and educational homogamy. American Sociological Review 56: 786-800.

Kaufmann, F.-X., 1988: Familie und Modernität. S. 391-415 in: Lüscher, K./Schultheis, F./Wehrspaun, M. (Hrsg.): Die ,postmoderne' Familie. Konstanz: Universitätsverlag.

Kohli, M., 1985: Die Institutionalisierung des Lebenslaufs. Kölner Zeitschrift für Soziologie und Sozialpsychologie 37: 1-29.

Kohli, M., 1989: Institutionalisierung und Individualisierung der Erwerbsbiographie. S. 249-278 in: Brock, Ditmar et al. (Hrsg.): Subjektivität im gesellschaftlichen Wandel. Weinheim: Juventa/DJI.

Lehrer, E.L./Kawasaki, S., 1985: Child care arrangements and fertility: an analysis of two-earner households. Demography 22: 499-513.

Linde, H., 1984: Theorie der säkularen Nachwuchsbeschränkung 1800 bis 2000 . Frankfurt/New York: Campus.

Luker, K., 1975: Taking chances. Berkeley: University of California Press.

Macfarlane, A., 1986: Marriage and love in England. Modes of reproduction 1300-1840. Oxford: Blackwell.

Mare, R.D., 1991: Five decades of educational assortative mating. American Sociological Review 56: 1532.

Mason, K.O./Kuhlthau, K., 1988: Determinants of child care among mothers of preschool-aged children. Research Reports No. 88-126, Population Studies Center, University of Michigan, Ann Arbor.

Mayer, K.U./Blossfeld, H.-P. (1990): Die gesellschaftliche Konstruktion sozialer Ungleichheit im Lebensverlauf. S. $297-318$ in: Berger, P.A./Hradil, S. (Hrsg.): Lebenslagen, Lebensläufe, Lebensstile (Sonderband 7 der Sozialen Welt). Göttingen: Schwartz.

McClosky, H./Zaller, J., 1984: The American ethos. Public attitudes toward capitalism and democracy. Cambridge, Mass.: Harvard University Press.

McLaughlin, S.D. et al., 1988: The changing lives of American women. Chapel Hill/London: The University of North Carolina Press.

Menaghan, E.G./Parcel, T.L., 1990: Parental employment and family life. Research in the 1980s. Journal of Marriage and the Family 52: 1079-1098.

Menken, J.A., 1985: Age and fertility: How late can you wait? Demography 22: 469-483.

Meyer, J.W., 1986: Myths of socialization and personality. S. 208-221 in: Heller, Th.C./Sosna, M./Wellbery, D.E. (eds.): Reconstructing individualism. Autonomy, individuality, and the self in Western thought. Stanford: Stanford University Press 
Meyer, Th., 1992: Modernisierung der Privatheit. Differenzierungs- und Individualisierungsprozesse des familialen Zusammenlebens. Westdeutscher Verlag.

Miller, B.C./Moore, K.A. 1990: Adolescent sexual behavior, pregnancy, and parenting: Research through the 1980s. Journal of Marriage and the Family. 52: 1025-1044.

Modell, J., 1989: Into one's own: From youth to adulthood in the Unites States 1920-1975. Berkeley: University of California Press.

Modell, J./Furstenberg, F.F./Strong, D., 1978: The timing of marriage in the transition to adulthood: Continuity and change, 1860-1975. S120-S150 in: Demos, J./Boocock, S.S. (eds.): Turning points: Historical and sociological essays on the family. Chicago: University of Chicago Press (Supplement to American Journal of Socioloy, Vol. 84).

Moen, P./Dempster-McClain, D.I., 1987: Employed parents: Role strain, work time, and preferences for working less. Journal of Marriage and the Family 49: 579-590.

Morgan, S.P., 1991: Late nineteenth- and early twentieth-century childlessness. American Journal of Sociology 97: 779-807.

Mosher, W.D., 1990: Contraceptive practice in the United States, 1982-1988. Family Planning Perspectives 22: $198-205$.

Mott, F.L., 1990: When is a father really gone? Paternalchild contact in father-absent homes. Demography 27: 499-517.

Nave-Herz, R., 1992: Ledige Mutterschaft: Eine alternative Lebensform? Zeitschrift für Sozialisationsforschung und Erziehungssoziologie 12: 219-232.

Popenoe, D., 1988: Disturbing the nest. Family change and decline in modern societies. New York: Aldine de Gruyter.

Rawlings, S.W., 1989: Single parents and their children. S. 13-25 in: U.S. Bureau of the Census, Studies in Marriage and the Family (Current Population Reports, Series P-23, No. 162). Washington, D.C.

Rindfuss, R.R./Morgan, S.P./Swicegood, G., 1988: First births in America. Changes in the timing of parenthood. Berkeley: University of California Press.

Rubin, L., 1976: Worlds of pain: Life in the workingclass family. New York: Basic Books.

Scanzoni, L.D./Scanzoni, J., 1988: Men, women, and change. A sociology of marriage and family. Third edition. New York: McGraw-Hill.

Scanzoni, J./Szinovacz, M., 1980: Family decisionmaking: A developmental sex-role model. Beverly Hills: Sage.

Seccombe, K., 1991: Assessing the costs and benefits of children: Gender comparison among childfree husbands and wifes. Journal of Marriage and the Family 53: 191-202.

Segalen, M., 1990: Die Familie. Geschichte, Soziologie, Anthropologie. Frankfurt: Campus.

Shorter, E., 1989: Einige demographische Auswirkungen des postmodernen Familienlebens. Zeitschrift für Bevölkerungswissenschaft 15: 221-233.
Sørensen, A., 1990: Towards an individualized lifc course? Contemporary Sociology 19: 297-299.

Sorrentino, C., 1990: The changing family in international perspective. Monthly Labor Review 113, no. 3: $41-58$.

Stacey, J., 1991: Zurück zur postmodernen Familie. Geschlechterverhältnisse, Verwandtschaft und soziale Schicht im Silicon Valley. Soziale Welt 42: 300-322.

Stack, C.B., 1974: All our kin. Strategies for survival in a black community. New York: Harper \& Row.

St. John, C., 1982: Race differences in age at first birth and the pace of subsequent fertility: Implications for the minority group status hypothesis. Demography 19: 301-314.

Sweet, J.A./Texeira, R.R., 1984: Breaking tradition: schooling, marriage, work, and childbearing in the lives of young women, 1960-1980. Center for Demography and Ecology Working Paper 84-13, Madison: University of Wisconsin.

Thornton, A., 1989: Changing attitudes toward family issues in the United States. Journal of Marriage and the Family 51: 873-894.

U.S. Bureau of the Census, 1961: Statistical Abstract of the United States. Washington, D.C.

U.S. Bureau of the Census, 1980: Statistical Abstract of the United States. Washington, D.C.

U.S. Bureau of the Census, 1989: Current Population Reports, Series P-20, No. 436, Fertility of American Women: June 1988. Washington, D.C.

U.S. Bureau of the Census, 1990: Statistical Abstract of the United States. Washington, D.C.

U.S. Dept. of Health and Human Services, 1990: Vital Statistics of the United States, 1988, Vol. I - Natality. Hyattsville, MD

Veevers, J.E., 1980: Childless by choice. Toronto: Butterworths.

Vinovskis, M.A., 1988: An „epidemic“ of adolescent pregnancy? Some historical and policy considerations. New York/Oxford: Oxford University Press.

Wahl, P., 1991: Wo der Mann noch ein Mann ist. S. 255264 in: Böhnisch, L. et al. (Hrsg.): Ländliche Lebenswelten. Fallstudien zur Landjugend. Weinheim/ München: Juventa/DJI.

Weisman, C.S./Teitelbaum, M.A., 1987: The work-family role system and physician productivity. Journal of Health and Social Behavior 28: 247-257.

Westoff, C.F./Ryder, N.B., 1977: The contraceptive revolution. Princeton, N.J.: Princeton University Press.

Weymann, A. (Hrsg.), 1989: Handlungsspielräume. Untersuchungen zur Individualisierung und Institutionalisierung von Lebensläufen in der Moderne. Stuttgart: Enke.

Wilkie, J.R., 1981: The trend toward delayed parenthood. Journal of Marriage and the Family 43: 583591.

Wilson, W.J., 1991: Studying inner-city social dislocations: the challenge of public agenda research. American Sociological Review 56: 1-14.

Wohlrab-Sahr, M., 1992: Institutionalisierung oder Individualisierung des Lebenslaufs? Anmerkungen zu einer festgefahrenen Debatte. Bios 5: 1-19. 
Yankelovich, D., 1987: New rules: Searching for selffulfillment in a world turned upside down. S. 103-106 in: Bellah, R.N. et al. (eds.), Individualism \& commitment in American life. Readings on the themes of Habits of the Heart. New York: Harper \& Row.

Zapf, W. et al., 1987: Individualisierung und Sicherheit. Untersuchungen zur Lebensqualität in der Bundesrepublik Deutschland. München: Beck.
Zelizer, V.A., 1985: Pricing the priceless child. The changing social value of children. New York: Basic Books.

Zelnik, M./Kantner, J.F, 1980: Sexual activity, contraceptive use and pregnancy among metropolitan-area teenagers: $1971-79$. Family Planning perspectives 12 : 230-237. 